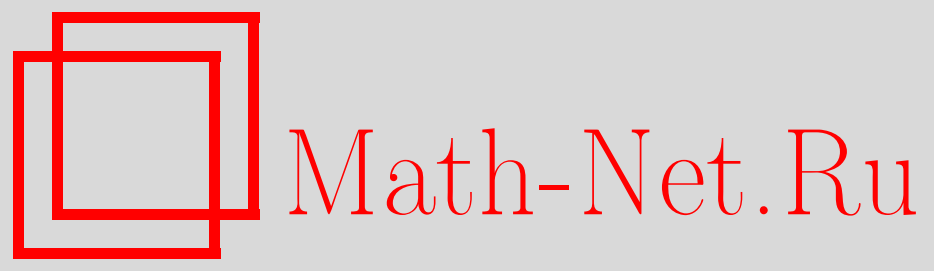

В. Э. Адлер, Необходимые условия интегрируемости для эволюционных уравнений на решетке, ТМФ, 2014, том 181, номер 2, 276-295

DOI: https://doi.org/10.4213/tmf8722

Использование Общероссийского математического портала Math-Net.Ru подразумевает, что вы прочитали и согласны с пользовательским соглашением http://www . mathnet.ru/rus/agreement

Параметры загрузки:

IP: 35.173 .137 .237

26 апреля 2023 г., $16: 47: 20$

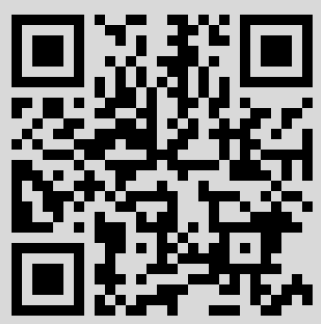




\title{
НЕОБХОДИМЫЕ УСЛОВИЯ ИНТЕГРИРУЕМОСТИ ДЛЯ ЭВОЛЮЦИОННЫХ УРАВНЕНИЙ НА РЕШЕТКЕ
}

\begin{abstract}
Изучена структура решений уравнения Лакса $D_{t}(G)=[F, G]$ для формальных рядов по степеням оператора сдвига. Показано, что если уравнение с заданным рядом $F$ степени $m$ допускает решение $G$ степени $k$, то оно допускает и решение $H$ степени $m$, такое что $H^{k}=G^{m}$. Это свойство применено для вывода необходимых условий интегрируемости для скалярных эволюционных цепочек.
\end{abstract}

Ключевые слова: цепочка типа Вольтерра, высшая симметрия, закон сохранения, тест на интегрируемость.

DOI: $10.4213 / \operatorname{tmf} 8722$

\section{1. ВВЕДЕНИЕ}

Хорошо известно, что характеристическим свойством интегрируемых уравнений является наличие бесконечных наборов высших симметрий и законов сохранения. Для двумерных эволюционных уравнений $\partial_{t}(u)=f[u]$, отсюда следует существование формальных операторных рядов $G, R$, удовлетворяющих уравнениям (см. раздел 3)

$$
D_{t}(G)=\left[f_{*}, G\right], \quad D_{t}(R)+f_{*}^{\dagger} R+R f_{*}=0 .
$$

Условия разрешимости (1) относительно коэффициентов $G, R$ служат необходимыми условиями интегрируемости. В работах Шабата и др. этот подход применялся для классификации интегрируемых уравнений, уравнений в частных производных [1]-[6] и дифференциально-разностных уравнений [2], [7]-[11]. Непрерывные и дискретные уравнения имеют много общего, но есть и различия.

В непрерывном случае $G$ и $R$ - это псевдодифференциальные операторы, т. е. ряды Лорана по обратным степеням полной производной $D$. Для однокомпонентных эволюционных уравнений коэффициенты рядов являются скалярными функциями от динамических переменных $u$. Очень важную роль в теории играет операция извлечения корня из таких рядов. Она определена для ряда $G=g_{k} D^{k}+g_{k-1} D^{k-1}+\cdots$

* Институт теоретической физики им. Л.Д. Ландау РАН, Черноголовка, Московская обл., Россия. E-mail: adler@itp.ac.ru 
общего вида, так как коэффициенты ряда $G^{1 / k}$ вычисляются при помощи явных алгебраических формул, не выводящих из рассматриваемого поля [12]. Благодаря этому изучение уравнений (1) сводится к случаю $\operatorname{deg} G=1, \operatorname{deg} R=0$, что существенно упрощает их анализ. В частности, условия интегрируемости переписываются в виде последовательности законов сохранения

$$
D_{t}\left(\rho_{j}\right)=D\left(\sigma_{j}\right), \quad j=0,1, \ldots,
$$

где $\rho_{j}$ явно определяются по $\rho_{i}, \sigma_{i}$ при $i<j$. Если левая часть $(2)$ лежит в образе $D$ (совпадающем с ядром вариационной производной), то можно найти $\sigma_{j}$ и переходить к следующему шагу.

В настоящей работе рассматривается случай уравнений на решетке

$$
\partial_{t}\left(u_{n}\right)=f\left(u_{n+m}, \ldots, u_{n-m}\right), \quad n \in \mathbb{Z},
$$

где ситуация несколько сложнее. Здесь $G, R$ - это ряды по степеням оператора сдвига $T$, и для ряда $G=g_{k} T^{k}+g_{k-1} T^{k-1}+\cdots$ общего вида корень $G^{1 / k}$ не определен. Это ясно уже из того, что для его существования нужно, чтобы старший коэффициент имел специальный вид $g_{k}=h T(h) \cdots T^{k-1}(h)$. В результате условия интегрируемости усложняются и, вообще говоря, не записываются в виде законов сохранения, а уравнения (1) теряют эффективность, поскольку степени рядов $G, R$ заранее неизвестны. Однако оказывается, что на самом деле условия интегрируемости не зависят от $k$. Целью статьи является доказательство того, что общий случай сводится к $\operatorname{deg} G=m, 0 \leqslant \operatorname{deg} R<m$.

В разделе 2 доказывается следующее утверждение: если разностное уравнение Лакса $D_{t}(G)=[F, G]$ с $\operatorname{deg} F=m$ допускает решение $G$ степени $k$, то существует и другое его решение $H=G^{m / k}$ степени $m$. Ключевая идея заключается в том, что коэффициенты $H$ можно вычислить по явным формулам, если использовать уравнения $D_{t}(H)=[F, H]$ и $H^{k}=G^{m}$ одновременно, после чего удается доказать, что оба уравнения выполняются по отдельности. Таким образом, извлечение корня в некотором ослабленном смысле все же возможно благодаря тому, что решения уравнения Лакса являются далеко не общими рядами и их коэффициенты уже имеют специальную структуру.

В разделах 3, 4 это наблюдение используется для формулировки необходимых условий интегрируемости для цепочек (3). При $m>1$ они остаются более сложными, чем в непрерывном случае, но вполне пригодны для тестирования заданного уравнения. Разрешение уравнения Лакса относительно коэффициентов $G$ сводится к проверке того, лежит ли заданное выражение в образе оператора вида $T^{m}-T^{j}(a) / a$, где $a$ - фиксированная функция, и к вычислению прообраза. В принципе обе задачи допускают алгоритмическое решение, что, однако, выходит за рамки настоящей статьи. Анализ условий в общем виде, для получения классификационных результатов или построения новых примеров, хотя бы в случае $m=2$, представляет собой весьма сложную задачу и также должен быть предметом отдельного исследования. Отметим, что все известные на данный момент примеры с $m>1$ сводятся (с точностью до подстановок типа Миуры) к цепочкам Богоявленского [13] и некоторым их обобщениям [14]-[16]. Здесь имеется отставание от непрерывного 
случая, для которого получен ряд классификационных результатов для уравнений типа Бюргерса порядков 2, 4 [17] и типа уравнения Кортевега-де Фриза порядков $3,5,7$ (см. ссылки в [6]).

Интегрируемые уравнения (3) при $m=1$ (цепочки типа Вольтерра) были проклассифицированы Ямиловым [7]. В этом случае необходимые условия интегрируемости имеют вид, аналогичный (2):

$$
D_{t}\left(\rho_{j}\right)=(T-1)\left(\sigma_{j}\right), \quad \rho_{j}-\bar{\rho}_{j}=(T-1)\left(s_{j}\right), \quad j=0,1, \ldots,
$$

где $\rho_{j}, \bar{\rho}_{j}$ определяются по $\rho_{i}, \bar{\rho}_{i}, \sigma_{i}, s_{i}$ при $i<j$. В работах [9], [10] вывод условий (4) основывался на предположении, что цепочка (3) обладает высшими симметриями порядков $k, k+1$, где $k$ сколь угодно велико, откуда следует существование ряда $G$ степени 1 . В то же время авторами отмечалось, что те же самые условия возникают и при рассмотрении ряда $G$ произвольной степени, хотя их вывод становится технически более сложным. Это полностью объясняется описанной выше процедурой извлечения корня, из которой следует также, что предположение о симметрии порядка $k+1$ излишне.

Что касается способа получения конкретных выражений для условий типа (2) или (4), то напомним, что плотности $\rho_{j}$ для непрерывных уравнений Лакса можно вычислять двумя способами: как вычеты дробных степеней $\operatorname{res} G^{j / k}[18]$ или как коэффициенты разложения по $\lambda$ для логарифмической производной от формальной функции Бейкера-Ахиезера ([19], идея восходит к построению производящей функции законов сохранения при помощи обращения преобразования Миуры [20]). Эквивалентность обоих определений установлена Уилсоном [19] и Флашкой [21]. В приложении к выводу необходимых условий интегрируемости техника, основанная на вычетах, подробно описана, например, в работах [1], [2]. Она требует более сложных вычислений, чем метод, основанный на разложении формальной $\psi$-функции [22], [23], [6]. Оба метода работают и в разностном случае [24], но только в предположении, что $\operatorname{deg} G=1$, что, как было отмечено выше, является существенной оговоркой.

В разделе 5 условия (4) выводятся при помощи разложения формальной функции $\psi$, что проще вычисления $\operatorname{Res} G^{j}$ и позволяет получить для плотностей явные компактные выражения в терминах полиномов Белла. Впрочем, как следует из результатов Ямилова [7], [10], для выделения списка интегрируемых цепочек при $m=1$ нужны лишь три простейших условия, которые нетрудно получить без всякой теории и при очень скромных предположениях о симметриях и законах сохранения. Это позволяет надеяться, что и в случае, скажем, $m=2$ классификация требует рассмотрения не слишком большого числа условий.

\section{2. ИЗВЛЕЧЕНИЕ КОРНЯ В РАЗНОСТНОМ СЛУЧАЕ}

Пусть $\mathcal{F}$ - поле локально аналитических функций от конечного числа динамических переменных $u_{n}, n \in \mathbb{Z}$, и оператор сдвига $T$ действует на функции из $\mathcal{F}$ по правилу

$$
T\left(a\left(u_{i}, \ldots, u_{j}\right)\right)=a\left(u_{i+1}, \ldots, u_{j+1}\right) .
$$

Правило $a T^{i} b T^{j}=a T^{i}(b) T^{i+j}$, распределенное по сложению, определяет умножение разностных операторов, при этом формальные ряды Лорана по отрицательным или 
положительным степеням $T$ образуют тела

$$
\mathcal{F}\left(\left(T^{-1}\right)\right)=\left\{\sum_{j<+\infty} a_{j} T^{j} \mid a_{j} \in \mathcal{F}\right\}, \quad \mathcal{F}((T))=\left\{\sum_{j>-\infty} a_{j} T^{j} \mid a_{j} \in \mathcal{F}\right\} .
$$

Все утверждения в этом разделе сформулированы для рядов из $\mathcal{F}\left(\left(T^{-1}\right)\right)$, для переноса на $\mathcal{F}((T))$ достаточно сделать переобозначение $u_{n} \rightarrow u_{-n}, T \rightarrow T^{-1}$.

Пусть $F=f_{m} T^{m}+\cdots \in \mathcal{F}\left(\left(T^{-1}\right)\right)$ - заданный ряд степени $m>0, D_{t}: \mathcal{F} \rightarrow \mathcal{F}-$ заданное эволюционное дифференцирование (т. е. коммутирующее с $T$; это обеспечивает то, что $D_{t}$ является также дифференцированием в $\left.\mathcal{F}\left(\left(T^{-1}\right)\right)\right)$. Рассмотрим уравнение Лакса

$$
D_{t}(G)=[F, G]
$$

и будем искать его решение в виде ряда $G \in \mathcal{F}\left(\left(T^{-1}\right)\right)$ степени $k>0$. В отличие от непрерывной ситуации, для ряда $G$ общего вида корень $G^{1 / k}$ не определен, поэтому изучение решений (или препятствий к их существованию) нельзя свести к случаю $k=1$. Тем не менее оказывается, что если решение $G$ существует, то его коэффициенты имеют некую специальную структуру, благодаря чему выполняются следующие свойства.

1. Уравнение (5) допускает другое решение $H$, степени $m$, такое что $H^{k}=G^{m}$. При этом $m$ не обязательно является минимальной положительной степенью решений. Таким образом, в дискретном случае извлечение корня возможно в некотором ослабленном смысле. Это свойство доказывается в теореме 1.

2. Среди решений степени $m$ можно выбрать такое, что $G=F+o(T)$ (т. е. $\operatorname{deg}(G-$ $F)<1)$. На первый взгляд, это очевидно, поскольку (5) дает те же уравнения для отрезка ряда $G_{>0}=g_{m} T^{m}+\cdots+g_{1} T$, что и уравнение $[F, G]=0$. Однако их общее решение может отличаться от $F_{>0}$ лишними постоянными параметрами, а последующие уравнения могут оказаться разрешимыми только при определенном выборе этих постоянных. В принципе может случиться так, что уравнения для всех коэффициентов $G$ разрешимы в $\mathcal{F}$ только при таких постоянных, что $G_{>0} \neq F_{>0}$. То, что этого не происходит, доказывается в следствии 1.

Прежде чем переходить к доказательствам, рассмотрим несколько конкретных формул для коэффициентов решений уравнения Лакса в двух простейших примеpax.

Пример 1: $m=1, k=2$. Пусть $F=f_{1} T+f_{0}+\cdots$ и уравнение (5) имеет решение $G=g_{2} T^{2}+g_{1} T+\cdots$. Можно ли найти решение $H$, такое что $H^{2}=G$ ? Выпишем несколько первых уравнений на коэффициенты $G$ :

$$
\begin{aligned}
0 & =f_{1} T\left(g_{2}\right)-T^{2}\left(f_{1}\right) g_{2}, \\
D_{t}\left(g_{2}\right) & =f_{1} T\left(g_{1}\right)-T\left(f_{1}\right) g_{1}+f_{0} g_{2}-T^{2}\left(f_{0}\right) g_{2}, \\
D_{t}\left(g_{1}\right) & =f_{1} T\left(g_{0}\right)-f_{1} g_{0}+f_{0} g_{1}-T\left(f_{0}\right) g_{1}+f_{-1} T^{-1}\left(g_{2}\right)-T^{2}\left(f_{-1}\right) g_{2} .
\end{aligned}
$$


Из первого уравнения находим $g_{2}=f_{1} T\left(f_{1}\right)$ (с точностью до постоянного множителя), после этого второе уравнение приводится к виду

$$
(T+1)\left(D_{t}\left(\ln f_{1}\right)\right)=(T-1)\left(\frac{g_{1}}{f_{1}}\right)-\left(T^{2}-1\right)\left(f_{0}\right),
$$

откуда следует, что существует функция $h_{0} \in \mathcal{F}$, такая что

$$
\frac{g_{1}}{f_{1}}=(T+1)\left(h_{0}\right), \quad D_{t}\left(\ln f_{1}\right)=(T-1)\left(h_{0}-f_{0}\right) .
$$

(Здесь используются свойства разностных операторов с постоянными коэффициентами: $\operatorname{ker}(T+1)=0, \operatorname{ker}(T-1)=\mathbb{C}$.) Подстановка в третье уравнение после несложных преобразований дает

$$
(T+1)\left(D_{t}\left(h_{0}\right)\right)=(T-1)\left(g_{0}-h_{0}^{2}\right)-\left(T^{2}-1\right)\left(f_{-1} T^{-1}\left(f_{1}\right)\right),
$$

откуда следует, что существует функция $h_{-1} \in \mathcal{F}$, такая что

$$
g_{0}=h_{0}^{2}+(T+1)\left(h_{-1} T^{-1}\left(f_{1}\right)\right), \quad D_{t}\left(h_{0}\right)=(T-1)\left(\left(h_{-1}-f_{-1}\right) T^{-1}\left(f_{1}\right)\right) .
$$

Собирая все вместе, получаем

$$
\begin{aligned}
G & =f_{1} T\left(f_{1}\right) T^{2}+f_{1}(T+1)\left(h_{0}\right) T+h_{0}^{2}+(T+1)\left(h_{-1} T^{-1}\left(f_{1}\right)\right)+o(1)= \\
& =\left(f_{1} T+h_{0}+h_{-1} T^{-1}\right)^{2}+o(1),
\end{aligned}
$$

что позволяет предположить, что корень действительно извлекается.

ПримеР 2: $m=2, k=2$. Пусть $F=f_{2} T^{2}+f_{1} T+\cdots$ и уравнение (5) имеет решение $G=f_{2} T^{2}+g_{1} T+\cdots$. Можно ли при этом положить $g_{1}=f_{1}$ ? Пусть $g_{1} \neq f_{1}$, тогда ряд $F-G$ имеет степень 1 , и, повторяя вычисления из предыдущего примера для уравнения $D_{t}(G)=[F-G, G]$, приходим к формулам

$$
G=\left(h_{1} T+h_{0}+h_{-1} T^{-1}\right)^{2}+o(1), \quad f_{1}-g_{1}=c h_{1}, \quad f_{2}=g_{2}=h_{1} T\left(h_{1}\right) .
$$

Таким образом, если считать доказанным, что $G=H^{2}$, то мы действительно можем перейти к решению $G+c H=f_{2} T^{2}+f_{1} T+\cdots$.

Наши вычисления имеют тот недостаток, что в них коэффициенты ряда $G^{1 / 2}$ находятся неявно, при помощи обращения операторов $T+1$ или $T-1$. Однако их можно получить и по явным формулам, если заранее известно, что искомый ряд существует. Идея заключается в том, что для извлечения корня нужно одновременно использовать оба уравнения $D_{t}(H)=[F, H]$ и $H^{2}=G$. Это приводит к рекуррентным соотношениям типа

$$
f_{1} T\left(h_{j}\right)-T^{j}\left(f_{1}\right) h_{j}=D_{t}\left(h_{j+1}\right)+\cdots, \quad f_{1} T\left(h_{j}\right)+T^{j}\left(f_{1}\right) h_{j}=g_{j+1}+\cdots,
$$

где правые части содержат коэффициенты $h_{1}, h_{0}, \ldots, h_{j+1}$, найденные на предыдущих шагах, и вычитая, получаем для $h_{j}$ явное выражение. После этого еще нужно 
убедиться, что выполняются оба уравнения. Это не очевидно, но правдоподобно, поскольку эти уравнения совместны в том смысле, что из них следует уравнение $D_{t}(G)=[F, G]$, которое выполнено по условию.

Для реализации этих наводящих соображений при доказательстве теоремы 1 нам понадобятся ряды с неавтономными коэффициентами. Рассмотрим расширение поля $\mathcal{F}$ в виде кольца $\widetilde{\mathcal{F}}$, элементами которого служат последовательности локально аналитических функций $a(n)=a\left(n ; u_{r_{n}}, \ldots, u_{s_{n}}\right), n \in \mathbb{Z}$, причем каждая функция в последовательности зависит от своего собственного конечного набора динамических переменных. Элементы из $\mathcal{F}$ отождествляются с последовательностями специального вида $a(n)=a\left(u_{n+r}, \ldots, u_{n+s}\right)$. По определению последовательности перемножаются почленно, а действие оператора $T$ заключается просто в сдвиге по $n$, т. е. $T^{k}(a(n))=a(n+k)$. Умножение в кольце

$$
\widetilde{\mathcal{F}}\left(\left(T^{-1}\right)\right)=\left\{\sum_{j<+\infty} a_{j}(n) T^{j} \mid a_{j}(n)=a_{j}\left(n ; u_{r_{j, n}}, \ldots, u_{s_{j, n}}\right) \in \widetilde{\mathcal{F}}\right\}
$$

определяется, как и раньше, правилом $a(n) T^{i} b(n) T^{j}=a(n) b(n+i) T^{i+j}$. Нетрудно видеть, что в этом расширении уравнение Лакса всегда разрешимо.

Лемма 1. Пусть $F(n)=f_{m}(n) T^{m}+\cdots \in \widetilde{\mathcal{F}}\left(\left(T^{-1}\right)\right), m \geqslant 1, u f_{m}(n) \not \equiv 0$ nрu всех $n$. Тогда для любой степени $k$ существует единственный ряд $G(n)=g_{k}(n) T^{k}+$ $\cdots \in \widetilde{\mathcal{F}}\left(\left(T^{-1}\right)\right)$, удовлетворяющий уравнению (5) и заданным начальным условиям $G(0), G(1), \ldots, G(m-1)$.

ДоКАЗАТЕЛЬСТво. Коэффициенты $g_{j}(n)$ вычисляются последовательно при $j=$ $k, k-1, \ldots$ У Уравнение для $g_{j}(n)$ возникает из $(5)$ в порядке $T^{j+m}$ и представляет собой рекуррентное соотношение вида

$$
f_{m}(n) g_{j}(n+m)-f_{m}(n+j) g_{j}(n)=\ldots,
$$

где в правую часть входят члены уже найденных последовательностей $g_{i}(n)$ с номерами $i>j$. Отсюда значения $g_{j}(n), n \in \mathbb{Z}$, определяются однозначно при заданных $g_{j}(0), \ldots, g_{j}(m-1)$.

ТЕОрема 1. Пусть рядъ $G, F \in \mathcal{F}\left(\left(T^{-1}\right)\right)$ удовлетворяют уравнению Лакса $D_{t}(G)=[F, G] u \operatorname{deg} F=m \geqslant 1, \operatorname{deg} G=k \geqslant 1$. Тогда существует ряд $H \in$ $\mathcal{F}\left(\left(T^{-1}\right)\right)$ степени $m$, единственньй с точностью до умножения на $1^{1 / k}$, такой чmo $D_{t}(H)=[F, H]$ u $H^{k}=G^{m}$.

ДокАзАтельство. Старший коэффициент ряда $H$ удовлетворяет уравнению

$$
f_{m} T^{m}\left(h_{m}\right)=T^{m}\left(f_{m}\right) h_{m}
$$

общее решение которого в $\mathcal{F}$ имеет вид $h_{m}=$ const $\cdot f_{m}$. Постоянная определяется с точностью до корня из единицы путем сравнения старших членов в равенстве $H^{k}=G^{m}$. 
Следующие коэффициенты будем строить в виде последовательностей $h_{j}(n)$ из кольца $\widetilde{\mathcal{F}}$, как в лемме 1 . Докажем методом индукции по $j$, что существуют единственные начальные данные $H(0), H(1), \ldots, H(m-1)$, при которых выполняются условия

$$
\left.\left(H^{k}-G^{m}\right)\right|_{n=0}=\cdots=\left.\left(H^{k}-G^{m}\right)\right|_{n=m-1}=0 .
$$

Допустим, что уже найдены коэффициенты $h_{m}(n), \ldots, h_{j+1}(n)$, такие что уравнение $D_{t}(H)=[F, H]$ выполняется вплоть до порядка $T^{m+j+1}$, а уравнения (6) выполняются вплоть до порядка $T^{m(k-1)+j+1}$. Уравнения для $h_{j}(n)$, возникающие в следующих порядках, запишем при помощи операторов $A(n)=f_{m}(n) T^{m}$ и $X(n)=h_{j}(n) T^{j}$ :

$$
\begin{gathered}
{[A(n), X(n)]=a(n) T^{m+j}, \quad n \in \mathbb{Z},} \\
A(n)^{k-1} X(n)+A(n)^{k-2} X(n) A(n)+\cdots+X(n) A(n)^{k-1}= \\
=b(n) T^{(k-1) m+j}, \quad n=0, \ldots, m-1,
\end{gathered}
$$

где $a(n), b(n)$ - некоторые многочлены от коэффициентов рядов $F, G$ и уже известных коэффициентов $h_{m}, \ldots, h_{j+1}$ (в том числе с производными по $t$ ). Легко видеть, что уравнение (8) при помощи (7) сводится к уравнениям вида

$$
k X(n) A(n)^{k-1}=c(n) T^{(k-1) m+j},
$$

T. e.

$$
k h_{j}(n) f_{m}(n+j) \cdots f_{m}(n+j+(k-2) m)=c(n), \quad n=0, \ldots, m-1,
$$

что однозначно определяет начальные данные для последовательности $h_{j}(n)$ и завершает индуктивный переход.

Для построенного решения ряд $H^{k}-G^{m}$ является решением уравнения Лакса с нулевыми начальными условиями, и согласно лемме 1 он тождественно равен нулю. Следовательно, уравнения (8) выполняются для всех $n \in \mathbb{Z}$, а не только для указанных.

Далее заметим, что многочлены $a(n), b(n)$, а следовательно, и $c(n)$, имеют при всех $n$ один и тот же вид, как функции от коэффициентов рядов $F, G, H$. Это означает, что если $h_{j}(n)=p\left[F, G, H_{>j}\right]$, где $p$ - функция от конечного числа переменных, то $h_{j}(n+1)=p\left[T(F), T(G), T\left(H_{>j}\right)\right]$. Так как все $f_{i}, g_{i} \in \mathcal{F}$ и $h_{m}=f_{m} \in \mathcal{F}$, методом индукции получаем, что все $h_{j} \in \mathcal{F}$.

ЗАмЕчАниЕ 1. Из доказательства следует, что для вычисления первых $r$ коэффициентов $H$ требуется знать ровно $r$ коэффициентов $G$ (не считая коэффициенты $F)$. Действительно, ряд $G$ используется только в начальных условиях (6), где число коэффициентов из обоих рядов совпадает в каждом порядке по $T$.

Нетрудно показать, что если уравнение Лакса (5) имеет хотя бы одно нетривиальное решение (т. е. отличное от $c T^{0}$ ), то его общее решение представляется в виде ряда с постоянными коэффициентами

$$
G=\sum_{j<+\infty} c_{j} H^{j},
$$


где $H$ - решение минимальной положительной степени $d$. Отсюда, в частности, следует, что любые два решения коммутируют, а из доказанной теоремы следует, что $d$ является делителем $m$.

СлеДСТвИЕ 1. Если уравнение $D_{t}(G)=[F, G]$ допускает нетривиальное решение из $\mathcal{F}\left(\left(T^{-1}\right)\right)$, то оно допускает и решение вида

$$
G=f_{m} T^{m}+\cdots+f_{1} T+g_{0}+g_{-1} T^{-1}+\cdots \in \mathcal{F}\left(\left(T^{-1}\right)\right)
$$

ДокАЗАТЕЛЬство. Из теоремы 1 следует существование некоторого решения $G$ степени $m$. Без потери общности будем считать, что старшие коэффициенты $G$ и $F$ совпадают, и пусть $\operatorname{deg}(F-G)=l$. Если $l \leqslant 0$, то утверждение доказано, рассмотрим случай $1 \leqslant l<m$.

Применив теорему 1 к уравнению $D_{t}(G)=[F-G, G]$, найдем ряд $H \in \mathcal{F}\left(\left(T^{-1}\right)\right)$ степени $l$, удовлетворяющий уравнениям $D_{t}(H)=[F-G, H]$. Ряд $H=h_{l} T^{l}+\cdots$ коммутирует с $G$ и $f_{l}-g_{l}=c h_{l}$. Отсюда следует, что ряд $G^{\prime}=G+c H$ удовлетворяет исходному уравнению и $\operatorname{deg}\left(F-G^{\prime}\right)<l$. Повторяя, при необходимости, это рассуждение еще несколько раз, приходим к решению, совпадающему с $F$ до члена $f_{1} T$ включительно.

Отметим, что уравнение Лакса в первых $m$ порядках по $T$ может допускать решение $G_{>0}$, отличающееся от $F_{>0}$ лишними параметрами, даже если минимальная степень истинного решения $G$ равна $m$. В такой ситуации эти параметры автоматически обращаются в нуль при разрешении уравнений для последующих коэффициентов $G$.

ПримеР 1. Рассмотрим цепочку

$$
u_{, t}=f[u]=u_{1} u^{2} u_{-1}\left(u_{2}-u_{-2}\right),
$$

связанную подстановкой $u_{1} u=v$ с модифицированной цепочкой Вольтерра на растянутой решетке $v_{, t}=v^{2}\left(v_{2}-v_{-2}\right)$. Эта замена действует также на высших симметриях, что гарантирует (см. раздел 3 ) разрешимость уравнения Лакса с

$$
F=f_{*}=u_{1} u^{2} u_{-1} T^{2}+u^{2} u_{-1}\left(u_{2}-u_{-2}\right) T+\cdots .
$$

Нетрудно проверить, что уравнение Лакса в первых двух порядках допускает решения как второй, так и первой степени:

$$
G_{>0}=F_{>0}+c u u_{-1} T, \quad H_{>-1}=u u_{-1} T+u_{1} u_{-1}-u u_{-2},
$$

причем $G_{>0}=\left(H^{2}+c H\right)_{>0}$. Однако уравнение для третьего коэффициента $H$ оказывается неразрешимым в $\mathcal{F}$. Для решения $G$ это означает, что постоянную $c$ придется положить равной нулю при вычислении четвертого коэффициента. 


\section{3. ФОРМАЛЬНАЯ СИММЕТРИЯ}

Напомним основные понятия симметрийного подхода применительно к скалярным эволюционным цепочкам

$$
\partial_{t}\left(u_{n}\right)=T^{n}\left(f\left(u_{m}, \ldots, u_{\bar{m}}\right)\right) .
$$

Для определенности аргументы функций будем упорядочивать по убыванию номеров. Кроме того, будем считать (применяя при необходимости отражение $u_{n} \rightarrow u_{-n}$ ), что

$$
f^{(m)} \neq 0, \quad f^{(\bar{m})} \neq 0, \quad m \geqslant 1, \quad m \geqslant \bar{m},
$$

где $f^{(j)}=\partial_{j}(f), \partial_{j}=\partial / \partial u_{j}$. Числа $m$ и $-\bar{m}$ будем называть соответственно $n о$ рядком и нижним порядком цепочки. Функции $a \in \mathcal{F}$ сопоставим эволюиионную производную $\nabla_{a}$ и оператор линеаризачии $a_{*}$ :

$$
\nabla_{a}=\sum_{j \in \mathbb{Z}} T^{j}(a) \partial_{j}, \quad a_{*}=\sum_{j \in \mathbb{Z}} a^{(j)} T^{j} \in \mathcal{F}\left[T, T^{-1}\right] .
$$

Для дифференцирования в силу уравнения (9) будем использовать также обозначение $D_{t}=\nabla_{f}$. Следующие тождества легко проверяются:

$$
\left[\nabla_{a}, T\right]=0, \quad(T(a))_{*}=T a_{*}, \quad \nabla_{a}(b)=b_{*}(a), \quad\left(\nabla_{a}(b)\right)_{*}=\nabla_{a}\left(b_{*}\right)+b_{*} a_{*} .
$$

Цепочка

$$
\partial_{\tau}\left(u_{n}\right)=T^{n}\left(g\left(u_{k}, \ldots, u_{\bar{k}}\right)\right)
$$

называется симметрией цепочки (9), если выполнено условие

$$
\nabla_{f}(g)=\nabla_{g}(f)
$$

тождественно по $u_{j}$. Оно означает, что потоки $\partial_{t}, \partial_{\tau}$ коммутируют (что гарантирует существование совместного общего решения $u_{n}(t, \tau)$ ). Цепочка считается интегрируемой, если она допускает симметрии сколь угодно высокого порядка. Применяя линеаризацию, перепишем последнее уравнение в операторном виде

$$
\nabla_{f}\left(g_{*}\right)=\nabla_{g}\left(f_{*}\right)+\left[f_{*}, g_{*}\right]
$$

более удобном для анализа. Отбрасывая член $\nabla_{g}\left(f_{*}\right)$, имеющий фиксированную степень по $T$, приходим к уравнению

$$
D_{t}(G)=\left[f_{*}, G\right],
$$

решения которого называются формальными симметриями цепочки (9).

Теорема 2. Если чепочка (9) допускает симметрии (10) со сколь угодно большим $k$, то уравнение (12) имеет решение $G \in \mathcal{F}\left(\left(T^{-1}\right)\right)$ вида

$$
G=f^{(m)} T^{m}+\cdots+f^{(1)} T+g_{0}+g_{-1} T^{-1}+\cdots .
$$


Если цепочка (9) с $\bar{m}<0$ допускает симметрии (10) со сколь угодно больиим - $\bar{k}$, то уравнение (12) имеет решение $\bar{G} \in \mathcal{F}((T))$ вида

$$
\bar{G}=f^{(\bar{m})} T^{\bar{m}}+\cdots+f^{(-1)} T^{-1}+\bar{g}_{0}+\bar{g}_{1} T+\cdots .
$$

ДокаЗАтельство. С учетом замены $u_{n} \rightarrow u_{-n}, T \rightarrow T^{-1}$ достаточно доказать первое утверждение. Ряд

$$
g_{*}=g^{(k)} T^{k}+\cdots+g^{(1)} T+o(T) \in \mathcal{F}\left(\left(T^{-1}\right)\right)
$$

удовлетворяет уравнению (5) в порядках $T^{k+m}, \ldots, T^{m+1}$. Применяя процедуру извлечения корня, описанную в теореме 1 , с учетом замечания 1 приходим к ряду $G \in \mathcal{F}\left(\left(T^{-1}\right)\right)$, такому что

$$
D_{t}(G)=\left[f_{*}, G\right]+o\left(T^{2 m-k+1}\right), \quad \operatorname{deg} G=m .
$$

При этом согласно следствию 1 можно считать $G_{>0}=\left(f_{*}\right)_{>0}($ если $k>m)$. Так как $k$ можно выбрать сколь угодно большим, то уравнение (5) разрешимо во всех порядках по $T$.

Условия разрешимости уравнения (12) относительно коэффициентов ряда $G$ или $\bar{G}$ служат необходимыми условиями интегрируемости. Формула (15) показывает, что из существования симметрии порядка $k \geqslant m+r$ следует выполнение $r$ условий для коэффициентов $g_{0}, \ldots, g_{-r+1}$. Младшие симметрии с $k \leqslant m$ не дают никаких условий, поскольку они "теряются" на фоне тривиальной симметрии с $g=f$, которой отвечает оператор $G=\left(f_{*}\right)_{>0}$. Аналогично, если существует симметрия нижнего порядка $-\bar{k}=-\bar{m}+r$, то выполняются условия разрешимости для коэффициентов $\bar{g}_{0}, \ldots, \bar{g}_{r-1}$ ряда $\bar{G}$. К сожалению, неизвестно, сколько условий нужно проверить, чтобы гарантировать существование хотя бы одной симметрии, пусть даже невысокого порядка. Тем не менее эти условия достаточно удобны и для тестирования, и для классификации, поскольку они выписываются непосредственно по правой части цепочки и их вид не зависит от реальных порядков симметрий, которые заранее неизвестны.

Первое условие и некоторое следствие из $m$-го условия имеют особенно простой вид. Используем следующее простое свойство:

$$
\operatorname{Res}[A, B] \in \operatorname{Im}(T-1), \quad \operatorname{Res} A:=\operatorname{coef}_{T^{0}} A, \quad A, B \in \mathcal{F}\left(\left(T^{-1}\right)\right)
$$

(действительно, $\left.\left[a T^{j}, b T^{-j}\right]=\left(T^{j}-1\right)\left(T^{-j}(a) b\right)\right)$.

УтВЕРЖДЕНИЕ 1. Если цепочка (9) допускает симметрию (10) порядка $k>2 m$, то существуют функиии $\sigma, \sigma_{1} \in \mathcal{F}$, такие что

$$
D_{t}\left(\ln f^{(m)}\right)=\left(T^{m}-1\right)(\sigma), \quad D_{t}\left(f^{(0)}+\sigma\right)=(T-1)\left(\sigma_{1}\right) .
$$

Если цепочка (9) допускает симметрию (10) с $\bar{k} \leqslant 2 \bar{m}<0$, то существуют функиии $\bar{\sigma}, \bar{\sigma}_{1} \in \mathcal{F}$, такие что

$$
D_{t}\left(\ln f^{(\bar{m})}\right)=\left(T^{\bar{m}}-1\right)(\bar{\sigma}), \quad D_{t}\left(f^{(0)}+\bar{\sigma}\right)=(T-1)\left(\bar{\sigma}_{1}\right) .
$$


ДокАЗАТЕЛЬСТво. Согласно теореме 2 уравнение (12) разрешимо в порядках $T^{2 m}, \ldots, T^{0}$, причем можно принять $G_{>0}=\left(f_{*}\right)_{>0}$. Применяя операцию Res к уравнению (12) и эквивалентному уравнению

$$
D_{t}(G) G^{-1}=f_{*}-G-G\left(f_{*}-G\right) G^{-1},
$$

получаем соответственно

$$
D_{t}\left(g_{0}\right) \in \operatorname{Im}(T-1), \quad \frac{D_{t}\left(f^{(m)}\right)}{f^{(m)}}=\left(T^{m}-1\right)\left(g_{0}-f^{(0)}\right),
$$

что равносильно (16). Формулы (17) выводятся аналогично.

Вообще, уравнение (12) в каждом порядке по $T$ имеет вид

$$
A_{j}\left(g_{j}\right)=b_{j}, \quad A_{j}=T^{m}-\frac{T^{j}(a)}{a}, \quad a=f^{(m)}, \quad j=0,-1,-2, \ldots
$$

где $b_{j}$ - известное выражение, содержащее коэффициенты $f_{*}$ и $g_{0}, \ldots, g_{j+1}$. Таким образом, тест на интегрируемость для заданной цепочки заключается в проверке, шаг за шагом, выполнено ли условие $b_{j} \in \operatorname{Im} A_{j}$. Если оно не выполняется, то цепочка неинтегрируема, если выполняется, то нужно вычислить $g_{j}$ и перейти к следующему условию. Заметив, что уравнение $A_{j+m}(g)=b$ сводится к $A_{j}(\tilde{g})=\tilde{b}$ в результате замены $g=T^{j}(a) \tilde{g}$, приходим к задаче о характеризации образа и вычислении прообраза для операторов

$$
T^{m}-1, \quad T^{m}-\frac{T(a)}{a}, \quad \ldots, \quad T^{m}-\frac{T^{m-1}(a)}{a}
$$

с заданной функцией $a$. В случае $m=1$ решение хорошо известно:

$$
\operatorname{Im}(T-1)=\operatorname{ker} \mathrm{E}, \quad \mathrm{E}=\frac{\delta}{\delta u}=\sum_{j \in \mathbb{Z}} T^{-j} \partial_{j},
$$

где $\mathrm{E}$ - оператор Эйлера или вариачионная производная, а прообраз $T-1$ можно найти, применяя разностную версию алгоритма интегрирования по частям или при помощи оператора гомотопии (см., например, [24], [25]). При $m>1$ задача также допускает конструктивное решение, хотя и более сложное (в частности, ответ зависит от того, лежит ли $\ln a$ в образе оператора $T^{m-d}+\cdots+T^{d}+1$, где $d$ - делитель $\left.m\right)$.

\section{4. ФОРМАЛЬНЫЙ ЗАКОН СОХРАНЕНИЯ}

Наибольший интерес представляет симметричный случай $\bar{m}=-m$, поскольку только у таких цепочек могут быть законы сохранения высокого порядка. Напомним, что функция $\rho \in \mathcal{F}$ называется плотностъю закона сохранения для цепочки (9), если существует функция $\sigma \in \mathcal{F}$, такая что

$$
\nabla_{f}(\rho)=(T-1)(\sigma)
$$


Плотность называется тривиальной, если $\rho \in \operatorname{Im}(T-1)$, и две плотности называются эквивалентными, если их разность тривиальна. Чтобы факторизовать тривиальные законы сохранения, применим к (18) оператор Эйлера, это дает уравнение для $r=$ $\mathrm{E}(\rho)=\rho_{*}^{\dagger}(1)$ :

$$
\nabla_{f}(r)+f_{*}^{\dagger}(r)=0
$$

где $\dagger: \mathcal{F}\left(\left(T^{ \pm 1}\right)\right) \rightarrow \mathcal{F}\left(\left(T^{\mp 1}\right)\right)$ обозначает сопряжение $\left(a T^{j}\right)^{\dagger}=T^{-j} a$. Еще раз применив линеаризацию, приходим к операторному уравнению

$$
\nabla_{f}\left(r_{*}\right)+f_{*}^{\dagger} r_{*}+r_{*} f_{*}+\sum_{m \geqslant i, j \geqslant \bar{m}} T^{-j}\left(r f^{(i, j)}\right) T^{i-j}=0 .
$$

Отметим, что оператор $r_{*}$ симметричен, $r_{*}=r_{*}^{\dagger}$. В частности, $r$ зависит от симметричного набора переменных: $r=r\left(u_{k}, \ldots, u_{-k}\right), r^{( \pm k)} \neq 0$. Число $k$ называется порядком закона сохранения. Нетрудно видеть, что степени по $T$ для четырех слагаемых в уравнении (19) равны соответственно $k, k-\bar{m}, k+m$ и $M \leqslant m-\bar{m}$. Отсюда следует, что цепочка (9) с $\bar{m} \neq-m$ не может иметь законов сохранения порядка $k>\min (m,-\bar{m})$. Итак, далее будем считать, что $\bar{m}=-m$, т. е. цепочка имеет вид

$$
\partial_{t}\left(u_{n}\right)=T^{n}\left(f\left(u_{m}, \ldots, u_{-m}\right)\right), \quad f^{( \pm m)} \neq 0
$$

Отбрасывая в (19) последнюю сумму, имеющую фиксированную степень по $T$, приходим к уравнению

$$
D_{t}(R)+f_{*}^{\dagger} R+R f_{*}=0
$$

решение которого в виде ряда из $\mathcal{F}\left(\left(T^{-1}\right)\right)$ или $\mathcal{F}((T))$ называется формальным законом сохранения для цепочки (20). Уравнение (21) инвариантно относительно сопряжения, поэтому можно ограничиться рядами из $\mathcal{F}\left(\left(T^{-1}\right)\right)$.

ТЕОРема 3. Пусть цепочка (20) допускает законы сохранения сколь угодно высокого порядка $k$. Тогда уравнение (12) имеет решения вида

$$
\begin{aligned}
& G=f^{(m)} T^{m}+\cdots+f^{(1)} T+g_{0}+g_{-1} T^{-1}+\cdots \in \mathcal{F}\left(\left(T^{-1}\right)\right), \\
& \bar{G}=f^{(-m)} T^{-m}+\cdots+f^{(-1)} T^{-1}+\bar{g}_{0}+\bar{g}_{1} T+\cdots \in \mathcal{F}((T)),
\end{aligned}
$$

а уравнение (21) имеет решение вида

$$
R=r_{l} T^{l}+r_{l-1} T^{l-1}+\cdots \in \mathcal{F}\left(\left(T^{-1}\right)\right), \quad 0 \leqslant l<m
$$

maкое что

$$
\bar{G}^{\dagger} R=-R G
$$

ДокАЗАТЕЛьство. Будем рассматривать операторы $r_{*}^{\prime}, r_{*}$, отвечающие законам сохранения порядков $k^{\prime}>k>m$, как ряды из $\mathcal{F}\left(\left(T^{-1}\right)\right)$. Из (19) следует, что эти ряды удовлетворяют уравнению $(21)$ в старших $k-m$ порядках по $T$. Извлекая 
корень из ряда $r_{*}^{-1} r_{*}^{\prime}$ степени $k^{\prime}-k>0$, приходим к ряду степени $m$, удовлетворяющему уравнению (12), также в $k-m$ старших порядках, т. е. с точностью до членов $o\left(T^{3 m-k+1}\right)$. Так как $k$ сколь угодно велико, уравнение $(12)$ разрешимо во всех порядках по $T$, и, учитывая следствие 1 , получаем для него решение вида (22).

Законы сохранения порядка $k=q m+l, 0 \leqslant l<m$, образуют бесконечное множество хотя бы при одном $l$. Для соответствующих рядов $r_{*}$ ряды $r_{*} G^{-q}$ имеют степень $l$ и удовлетворяют уравнению $(21)$ в старших $(q-1) m+l$ порядках, откуда следует существование решения $R(24)$.

Для построенных рядов $G, R \in \mathcal{F}\left(\left(T^{-1}\right)\right)$ ряд $\bar{G}=-\left(R G R^{-1}\right)^{\dagger} \in \mathcal{F}((T))$ также является решением уравнения (12). При этом согласно (21)

$$
\left(\bar{G}^{\dagger}\right)_{>0}=-R G_{>0} R^{-1}=-R\left(f_{*}\right)_{>0} R^{-1}=\left(f_{*}^{\dagger}\right)_{>0},
$$

откуда следует, что $\bar{G}_{<0}=\left(f_{*}\right)_{<0}$.

Сравнение с теоремой 2 (при $\bar{m}=-m$ ) показывает, что предположение о наличии бесконечного набора законов сохранения дает более жесткие по сравнению с высшими симметриями условия интегрируемости:

$\begin{array}{ccc}\text { законы сохранения } & \text { высшие симметрии } \\ \Downarrow & \Downarrow & \Downarrow, \bar{G}\end{array}$

Недостатком условий, которые вытекают из уравнения для $R$, является то, что степень $l=\operatorname{deg} R$ заранее неизвестна, поэтому для их проверки приходится применять перебор для $l=0, \ldots, m-1$. В частности, вместо утверждения 1 получаем

УТВЕРЖДЕНИЕ 2. Если цепочка (20) допускает два закона сохранения порядков $k^{\prime}>k>3 m$, то существуют функиии $\sigma, \sigma_{1}, s, s_{1} \in \mathcal{F}$ u целое $l, 0 \leqslant l<m$, такие чmo

$$
\begin{aligned}
D_{t}\left(\ln f^{(m)}\right) & =\left(T^{m}-1\right)(\sigma), & D_{t}\left(f^{(0)}+\sigma\right) & =(T-1)\left(\sigma_{1}\right), \\
\ln \frac{-T^{l}\left(f^{(m)}\right.}{f^{(-m)}} & =\left(T^{m}-1\right)(s), & D_{t}(s)+2 f^{(0)} & =(T-1)\left(s_{1}\right) .
\end{aligned}
$$

ДокАЗАтЕЛЬство. В обозначениях теоремы 3 ряды $G, \bar{G}, R$, построенные по $r_{*}^{\prime}, r_{*}$, удовлетворяют уравнениям $(12),(21)$ в старших $2 m+1$ порядках. Равенства (26) доказываются, как в утверждении 1. Первое соотношение (27) следует из $(21)$ в старшем порядке, с функцией $s=\ln \left(f^{(-m)} r_{l}\right)$. Умножив (21) на $R^{-1}$ и применив операцию Res, получаем $D_{t}\left(\ln r_{l}\right)+2 f_{0} \in \operatorname{Im}(T-1)$, что равносильно второму соотношению (27). 


\section{5. ЦЕПОЧКИ ПЕРВОГО ПОРЯДКА}

Условия интегрируемости принимают особенно простой вид для цепочек первого порядка

$$
\partial_{t}\left(u_{n}\right)=T^{n}\left(f\left(u_{1}, u, u_{-1}\right)\right), \quad f^{( \pm 1)} \neq 0 .
$$

В этом случае уравнение Лакса для $G=f^{(1)} T+g_{0}+g_{-1} T^{-1}+\cdots$ оказывается эквивалентным последовательности законов сохранения (возможно, тривиальных), заданной определенными рекуррентными соотношениями. Для их записи нам понадобятся многочлены

$$
\begin{gathered}
P_{0}=1, \quad P_{1}\left(x_{1}\right)=x_{1}, \quad P_{2}\left(x_{1}, x_{2}\right)=x_{2}+\frac{x_{1}^{2}}{2}, \\
P_{3}\left(x_{1}, x_{2}, x_{3}\right)=x_{3}+x_{1} x_{2}+\frac{x_{1}^{3}}{6}, \quad \ldots,
\end{gathered}
$$

определяемые производящей функцией

$$
P_{0}[x]+P_{1}[x] \lambda+P_{2}[x] \lambda^{2}+\cdots=e^{x_{1} \lambda+x_{2} \lambda^{2}+x_{3} \lambda^{3}+\cdots} .
$$

Эти многочлены, хорошо известные в теории представлений бесконечномерных алгебр Ли (см., например, [26], [27]), связаны с полными экспоненциальными полиномами Белла $Y_{k}[28]$ заменой $k ! P_{k}\left(x_{1}, \ldots, x_{k}\right)=Y_{k}\left(x_{1}, \ldots, k ! x_{k}\right)$.

Чтобы переписать уравнение $D_{t}(G)=\left[f_{*}, G\right]$ в виде законов сохранения, воспользуемся тем, что оно служит условием совместности для уравнений

$$
G(\psi)=\lambda \psi, \quad D_{t}(\psi)=f_{*}(\psi)
$$

и рассмотрим разложения по $\lambda$ для отношений

$$
p=\frac{T(\psi)}{\psi}, \quad q=\frac{D_{t}(\psi)}{\psi}
$$

Это приводит к уравнениям

$$
\begin{gathered}
f^{(1)} p+g_{0}-\lambda+\frac{g_{-1}}{T^{-1}(p)}+\frac{g_{-2}}{T^{-1}(p) T^{-2}(p)}+\cdots=0, \\
q=f^{(1)} p+f^{(0)}+\frac{f^{(-1)}}{T^{-1}(p)}, \\
\frac{D_{t}(p)}{p}=(T-1)(q) .
\end{gathered}
$$

Нетрудно видеть, что уравнение (31) определяет обратимую замену между коэффициентами рядов $G$ и $p=p_{-1} \lambda+p_{0}+p_{1} \lambda^{-1}+\cdots$ :

$$
p_{-1}=\frac{1}{f^{(1)}}, \quad p_{0}=-\frac{g_{0}}{f^{(1)}}, \quad p_{1}=-\frac{g_{-1} T^{-1}\left(f^{(1)}\right)}{f^{(1)}}, \quad \ldots,
$$


такую что $G \in \mathcal{F}\left(\left(T^{-1}\right)\right)$, тогда и только тогда, когда $p \in \mathcal{F}\left(\left(\lambda^{-1}\right)\right)$. При этом из (32) следует, что $q=\lambda-\sigma_{0}-\sigma_{1} \lambda^{-1}-\ldots \in \mathcal{F}\left(\left(\lambda^{-1}\right)\right)$, а решение уравнений (29) (формальная функция Бейкера-Ахиезера) строится путем интегрирования уравнений (30) в виде ряда

$$
\psi(n)=a(n) \lambda^{n}\left(1+a_{1}(n) \lambda^{-1}+a_{2}(n) \lambda^{-2}+\cdots\right)
$$

с коэффициентами в некотором расширении поля $\mathcal{F}$. После еще одной замены

$$
p=\frac{\lambda}{f^{(1)}} e^{-\rho_{1} \lambda^{-1}-\rho_{2} \lambda^{-2}-\cdots}
$$

уравнения (32) и (33) сводятся к соотношениям

$$
\begin{gathered}
D_{t}\left(\rho_{j}\right)=(T-1)\left(\sigma_{j}\right), \quad j \geqslant 0, \\
\rho_{0}=\ln f^{(1)}, \quad \rho_{1}=f^{(0)}+\sigma_{0}, \\
P_{j+1}[-\rho]+f^{(-1)} T^{-1}\left(f^{(1)} P_{j-1}[\rho]\right)+\sigma_{j}=0, \quad j>0 .
\end{gathered}
$$

Существование формальной симметрии $G \in \mathcal{F}\left(\left(T^{-1}\right)\right)$ эквивалентно разрешимости уравнений $(35)$ относительно $\sigma_{j} \in \mathcal{F}$, причем плотности $\rho_{j+1}$ явно находятся из (37):

$$
\begin{aligned}
& \rho_{2}=f^{(-1)} T^{-1}\left(f^{(1)}\right)+\frac{1}{2} \rho_{1}^{2}+\sigma_{1}, \\
& \rho_{3}=f^{(-1)} T^{-1}\left(f^{(1)} \rho_{1}\right)+\rho_{1} \rho_{2}-\frac{1}{6} \rho_{1}^{3}+\sigma_{2}, \\
& \rho_{4}=f^{(-1)} T^{-1}\left(f^{(1)}\left(\rho_{2}+\frac{1}{2} \rho_{1}^{2}\right)\right)+\rho_{1} \rho_{3}+\frac{1}{2} \rho_{2}^{2}-\frac{1}{2} \rho_{1}^{2} \rho_{2}+\frac{1}{24} \rho_{1}^{4}+\sigma_{3}, \quad \ldots .
\end{aligned}
$$

Чтобы записать в аналогичном виде следствия из существования второй формальной симметрии $\bar{G} \in \mathcal{F}((T))$, рассмотрим функцию $\bar{\psi}$, удовлетворяющую уравнениям

$$
\bar{G}^{\dagger}(\bar{\psi})=-\lambda \bar{\psi}, \quad D_{t}(\bar{\psi})=-f_{*}^{\dagger}(\bar{\psi})
$$

Как и раньше, нетрудно проверить, что отношения

$$
\bar{p}=\frac{T(\bar{\psi})}{\bar{\psi}}, \quad \bar{q}=\frac{D_{t}(\bar{\psi})}{\bar{\psi}}
$$

имеют разложения по $\lambda$ вида

$$
\bar{p}=-\frac{\lambda}{T\left(f^{(-1)}\right)} e^{-\bar{\rho}_{1} \lambda^{-1}-\bar{\rho}_{2} \lambda^{-2}-\cdots}, \quad \bar{q}=\lambda-\bar{\sigma}_{0}-\bar{\sigma}_{1} \lambda^{-1}-\cdots
$$


с коэффициентами $\bar{\rho}_{j}, \bar{\sigma}_{j} \in \mathcal{F}$ и удовлетворяют уравнениям

$$
\bar{q}=-T\left(f^{(-1)}\right) \bar{p}-f^{(0)}-\frac{T^{-1}\left(f^{(1)}\right)}{T^{-1}(\bar{p})}, \quad \frac{D_{t}(\bar{p})}{\bar{p}}=(T-1)(\bar{q}) .
$$

Отсюда для функций $\bar{\rho}_{j}, \bar{\sigma}_{j}$ следуют те же рекуррентные соотношения (35), (37), что и для $\rho_{j}, \sigma_{j}$, но с начальными условиями

$$
\bar{\rho}_{0}=\ln \left(-T\left(f^{(-1)}\right)\right), \quad \bar{\rho}_{1}=-f^{(0)}+\bar{\sigma}_{0}
$$

вместо (36). Если цепочка (28) допускает к тому же формальный закон сохранения $R=r_{0}+r_{-1} T^{-1}+\cdots \in \mathcal{F}\left(\left(T^{-1}\right)\right)$, то из уравнений $(21),(25)$ следует, что можно принять $\bar{\psi}=R(\psi)$. Тогда ряд

$$
s=\ln \frac{\bar{\psi}}{\psi}=s_{0}+s_{1} \lambda^{-1}+\cdots \in \mathcal{F}\left(\left(\lambda^{-1}\right)\right)
$$

удовлетворяет уравнениям

$$
\ln \bar{p}-\ln p=(T-1)(s), \quad D_{t}(s)=\bar{q}-q,
$$

т. е. обе последовательности плотностей связаны соотношениями

$$
\rho_{j}-\bar{\rho}_{j}=(T-1)\left(s_{j}\right), \quad j \geqslant 0 .
$$

При этом функции $\bar{\sigma}_{j}=\sigma_{j}-D_{t}\left(s_{j}\right)$ можно не рассматривать, так как $\bar{\rho}_{j}$ находятся из рекуррентных соотношений

$$
\begin{gathered}
\bar{\rho}_{0}=\ln \left(-T\left(f^{(-1)}\right)\right), \quad \rho_{1}-\bar{\rho}_{1}=2 f^{(0)}+D_{t}\left(s_{0}\right), \\
P_{j+1}[-\bar{\rho}]-P_{j+1}[-\rho]+f^{(-1)} T^{-1}\left(f^{(1)}\left(P_{j-1}[\bar{\rho}]-P_{j-1}[\rho]\right)\right)=D_{t}\left(s_{j}\right), \quad j>0 .
\end{gathered}
$$

Как уже отмечалось в разделе 1, для выделения интегрируемых цепочек (28) хватает согласно [7] всего лишь трех простейших условий (одного условия типа (35) и двух условий типа (39), ср. также с утверждением 2), которые можно переписать в виде

$$
\begin{gathered}
D_{t}\left(\ln f^{(1)}\right) \in \operatorname{Im}(T-1) \\
\ln \frac{-f^{(1)}}{f^{(-1)}}=(T-1)(s), \quad D_{t}(s)+2 f^{(0)} \in \operatorname{Im}(T-1) .
\end{gathered}
$$

Их можно вывести из предположений, что цепочка допускает симметрию порядка $k \geqslant 2$ и закон сохранения порядка $k^{\prime} \geqslant 3$ или что она допускает два закона сохранения порядков $k^{\prime}>k \geqslant 3$. Анализ этих условий представляет собой довольно трудоемкую задачу и приводит к конечному списку цепочек. Перебором можно 
проверить, что все они обладают бесконечным набором высших симметрий (и законов сохранения, за исключением некоторых вырожденных случаев типа линейной цепочки $\partial_{t}\left(u_{n}\right)=u_{n+1}-u_{n-1}$, у которой нет законов сохранения выше нулевого порядка, но уравнение (21) допускает решение $R=1$ ).

Для полноты картины приведем утверждение об эквивалентности построенных законов сохранения (35) со стандартным определением через вычеты степеней формальной симметрии. Доказательство в основном следует Купершмидту (гл. IX.3 в [24]) и является перенесением на дискретный случай доказательства из работы Флашки [21].

УТВЕРЖДЕНИЕ 3. Пусть цепочка (28) допускает формалъную симметрию $G=$ $f^{(1)} T+\cdots \in \mathcal{F}\left(\left(T^{-1}\right)\right)$ и величины $\rho_{j}$ определены соотношениями $(31),(34)$, тогда

$$
\operatorname{Res} G^{j}-j \rho_{j} \in \operatorname{Im}(T-1), \quad j=1,2, \ldots
$$

ДокАзАтЕЛЬСтво. Из формул (29), (30) для функции $\psi$ имеем

$$
T(\psi)=p \psi=\sum_{j} p_{j} \lambda^{-j} \psi=\sum_{j} p_{j} G^{-j}(\psi)
$$

откуда следует тождество

$$
T=p_{-1} G+p_{0}+p_{1} G^{-1}+p_{2} G^{-2}+\cdots
$$

эквивалентное соотношению (31) между рядами $G$ и $p$. Введем обозначения

$$
G_{j}=\left(G^{j}\right)_{\geqslant 0}, \quad A_{j}=\left(G^{j}\right)_{<0}, \quad \epsilon_{j}=\operatorname{coef}_{T^{-1}} G^{j}
$$

(в частности, $\left.\epsilon_{-1}=1 / T^{-1}\left(f^{(1)}\right), \epsilon_{0}=0\right)$. Умножая (41) справа на $G^{j}$ и отбрасывая отрицательные степени $T$, получаем

$$
T G_{j}+T\left(\epsilon_{j}\right)=p_{-1} G_{j+1}+p_{0} G_{j}+\cdots+p_{j} G_{0}, \quad j=-1,0,1, \ldots,
$$

что равносильно равенству (разностная версия формулы Чередника [29])

$$
(T-p) \mathcal{G}=-T(E)
$$

для производящих функций

$$
\mathcal{G}=1+\lambda^{-1} G_{1}+\lambda^{-2} G_{2}+\cdots, \quad E=\epsilon_{-1} \lambda+\epsilon_{0}+\epsilon_{1} \lambda^{-1}+\cdots
$$


Применяя операцию Res, получаем

$$
\operatorname{Res} \mathcal{G}=1+\lambda^{-1} \operatorname{Res} G+\lambda^{-2} \operatorname{Res} G^{2}+\cdots=\frac{T(E)}{p} .
$$

Далее, обозначим

$$
\alpha_{j}=\frac{A_{j}(\psi)}{\psi}=\frac{\epsilon_{j}}{T^{-1}(p)}+\frac{a_{j,-2}}{T^{-1}(p) T^{-2}(p)}+\cdots=\epsilon_{j} T^{-1}\left(f^{(1)}\right) \lambda^{-1}+\cdots
$$

тогда

$$
\frac{G_{j}(\psi)}{\psi}=\lambda^{j}-\alpha_{j}, \quad \frac{T G_{j}(\psi)}{\psi}=p\left(\lambda^{j}-T\left(\alpha_{j}\right)\right) .
$$

Применим тождество $(42)$ к $\psi(\mu)$ и разделим на $\psi(\mu)$ :

$$
\begin{aligned}
-T(E(\lambda)) & =(T-p(\lambda)) \frac{\mathcal{G}(\lambda)(\psi(\mu))}{\psi(\mu)}= \\
& =\sum_{j \geqslant 0} \lambda^{-j}\left[p(\mu)\left(\mu^{j}-T\left(\alpha_{j}(\mu)\right)\right)-p(\lambda)\left(\mu^{j}-\alpha_{j}(\mu)\right)\right]= \\
& =\frac{\lambda(p(\mu)-p(\lambda))}{\lambda-\mu}+\sum_{j \geqslant 0} \lambda^{-j}\left[p(\lambda) \alpha_{j}(\mu)-p(\mu) T\left(\alpha_{j}(\mu)\right)\right] .
\end{aligned}
$$

Разделим на $-p(\lambda)$ и перейдем к пределу $\mu \rightarrow \lambda$, получим

$$
\frac{T(E)}{p}=\lambda \partial_{\lambda}(\ln p)+\sum_{j \geqslant 0} \lambda^{-j}(T-1)\left(\alpha_{j}\right)
$$

Утверждение следует из сравнения с (34) и (43).

В заключение отметим, что аналог замены $p=T(\psi) / \psi$ существует и для цепочек порядка $m$ : можно показать, что уравнение $G(\psi)=\lambda \psi$ с рядом $G \in \mathcal{F}\left(\left(T^{-1}\right)\right)$ степени $m$ эквивалентно уравнению

$$
\left(T^{m}-p_{m-1} T^{m-1}-\cdots-p_{0}\right)(\psi)=0, \quad p_{j} \in \mathcal{F}\left(\left(\lambda^{-1}\right)\right) .
$$

В матричных обозначениях уравнения (29) принимают вид

$$
T(\Psi)=P \Psi, \quad D_{t}(\Psi)=Q \Psi
$$

где

$$
\Psi=\left(\begin{array}{c}
\psi \\
\vdots \\
T^{m-1}(\psi)
\end{array}\right), \quad P=\left(\begin{array}{ccccc}
0 & 1 & 0 & \ldots & 0 \\
0 & 0 & 1 & \ddots & \vdots \\
\vdots & \vdots & \ddots & \ddots & 0 \\
0 & 0 & \ldots & 0 & 1 \\
p_{0} & p_{1} & p_{2} & \ldots & p_{m-1}
\end{array}\right)
$$


и элементы матрицы $Q$ выражаются через $f^{(j)}$ и $p_{j}$. Уравнение $(33)$ заменяется на уравнение

$$
D_{t}(P)=T(Q) P-P Q
$$

откуда, в частности, следует, что $\ln p_{0}$ служит производящей функцией для законов сохранения. Однако в приложении к выводу условий интегрируемости это уравнение приводит к довольно громоздким формулам и, по-видимому, не дает преимуществ по сравнению с прямолинейным разрешением уравнения Лакса для формальной симметрии.

Благодарности. Большое значение для автора имели обсуждения данной темы с Р.И. Ямиловым. Условия (26), (27) в несколько более слабой форме были получены Р. И. Ямиловым в 2008 г. исходя из предположения о существовании формальной симметрии произвольной степени [30]. Настоящее исследование было поддержано РФФИ (грант № 13-01-00402_а) и Программой SFB/TR 109 "Discretization in Geometry and Dynamics".

\section{Список литературы}

[1] V. V. Sokolov, A. B. Shabat, Sov. Sci. Rev. Sect. C, 4 (1984), 221-280.

[2] А. В. Михайлов, А. Б. Шабат, Р. И. Ямилов, УМН, 42:4(256) (1987), 3-53.

[3] В. В. Соколов, УМН, 43:5(263) (1988), 133-163.

[4] A. V. Mikhailov, A. B. Shabat, V. V. Sokolov, "The symmetry approach to classification of integrable equations", What is Integrability?, ed. V.E. Zakharov, Springer, Berlin, 1991, $115-184$.

[5] A. B. Shabat, A. V. Mikhailov, "Symmetries - test of integrability", Important Developments in Soliton Theory, eds. A. S. Fokas, V. E. Zakharov, Springer, Berlin, 1993, 355-372.

[6] А.Г. Мешков, В. В. Соколов, Уфимск. матем. журн., 4:3 (2012), 104-154, arXiv: 1302.6010 .

[7] Р. И. Ямилов, УМН, 38:6(234) (1983), 155-156.

[8] А. Б. Шабат, Р. И. Ямилов, Алгебра и анализ, 2:2 (1990), 183-208.

[9] D. Levi, R. I. Yamilov, J. Math. Phys., 38:12 (1997), 6648-6674.

[10] R. I. Yamilov, J. Phys. A, 39:45 (2006), R541-R623.

[11] V.E. Adler, J. Phys. A, 41:14 (2008), 145201, 14 pp.

[12] I. Schur, "Über vertauschbare lineare Differentialausdrücke", Sitzungsber. Berl. Math. Ges., 4 (1905), 2-8.

[13] О. И. Богоявленский, УМН, 46:3(279) (1991), 3-48.

[14] V.E. Adler, V. V. Postnikov, J. Phys. A, 44:41 (2011), 415203, 17 pp.

[15] R. N. Garifullin, R. I. Yamilov, J. Phys. A, 45:34 (2012), 345205, 23 pp.

[16] Р. Н. Гарифуллин, А. В. Михайлов, Р. И. Ямилов, ТМФ, 180:1 (2014), 17-34.

[17] С. И. Свинолупов, ТМФ, 65:2 (1985), 303-307.

[18] И. М. Гельфанд, Л. А. Дикий, Функи. анализ и его прил., 10:4 (1976), 13-29.

[19] G. Wilson, Quart. J. Math., 32:4 (1981), 491-512.

[20] R. M. Miura, C. S. Gardner, M. D. Kruskal, J. Math. Phys., 9:8 (1968), 1204-1209.

[21] H. Flaschka, Quart. J. Math., 34:1 (1983), 61-65.

[22] H. H. Chen, Y. C. Lee, C. S. Liu, Phys. Scr., 20:3-4 (1979), 490-492. 
[23] A. G. Meshkov, Inverse Probl., 10:3 (1994), 635-653.

[24] B.A. Kupershmidt, Discrete Lax Equations and Differential-Difference Calculus, Astérisque, 123, Sociéte mathématique de France, Paris, 1985.

[25] P. E. Hydon, E. L. Mansfield, Found. Comput. Math., 4:2 (2004), 187-217.

[26] M. Jimbo, T. Miwa, Publ. Res. Inst. Math. Sci., 19:3 (1983), 943-1001.

[27] V. G. Kac, A. K. Raina, Bombay Lectures on Highest Weight Representations of Infinite Dimensional Lie Algebras, Advanced Series in Mathematical Physics, 2, World Sci., Singapore, 1987.

[28] L. Comtet, Advanced Combinatorics. The Art of Finite and Infinite Expansions, D. Reidel, Dordrecht, 1974.

[29] И. В. Чередник, Функи. анализ и его прил., 12:3 (1978), 45-54.

[30] R. I. Yamilov, "Symmetries as integrability criteria", Continuous Symmetries and Integrability of Discrete Equations, eds. D. Levi, P. Winternitz, R. I. Yamilov, in preparation.

Поступила в редакцию 1.06.2014 